\title{
How medical trainees actually spend their time during clinical shifts
}

\author{
n Cite as: CMAJ 2019 June 10;191:E645-6. doi: 10.1503/cmaj.109-5759
}

Posted on cmajnews.com on May 21, 2019.

M edical trainees are spending more time in front of screens and less engaging with patients compared to two decades ago, according to recent research. In a study published in JAMA Internal Medicine, observers logged the tasks of 80 medical trainees.

Interacting with an electronic health record (EHR) accounted for $43 \%$ of a resident's shift; $21 \%$ was spent on rounds, $13 \%$ on direct patient care and $7 \%$ on educational activities. The study was unique in that it also measured how often trainees multitasked $-23 \%$ of direct patient care and $28 \%$ of educational activities occurred during indirect patient care (while communicating with team members, for example).

Dr. Michael Arget, president of Resident Doctors of Canada, said the results aren't surprising. "I think, definitely, we should be spending more time interacting with patients and examining patients," he said. But he acknowledged that medicine has changed, and more doctors are working in interdisciplinary teams, which involves keeping track of updates from other providers and communicating with them via an EHR.

"We're moving into a landscape now with specialized medicine where no one can know everything there is ... You may be coordinating with the pathologist, the respiratory therapist, the radiologist, and that requires paperwork and making calls to make sure that plan is consistent."

Given this new normal, Arget wishes electronic systems were more optimized. "It takes me at least a minute or two to log into the $[E H R]$ on a good day, when the computer I'm at is actually working," he said. "I have to put my password in five to six different times."

As for multitasking, Arget thinks that's on the rise. He said many internal medicine residents are managing $6-10$ patients, usually with complex comorbidities. "While you're trying to see those patients and look at their notes, the paging is incessant from other units in the hospital or elsewhere to ask for information." He worries the multitasking stress is contributing to burnout and recommends that more effort be made to reduce interruptions, such as having "no paging zones" when residents are learning important new skills.

There was a time when residents spent more of their shifts interacting with patients. A study from 1994 reported

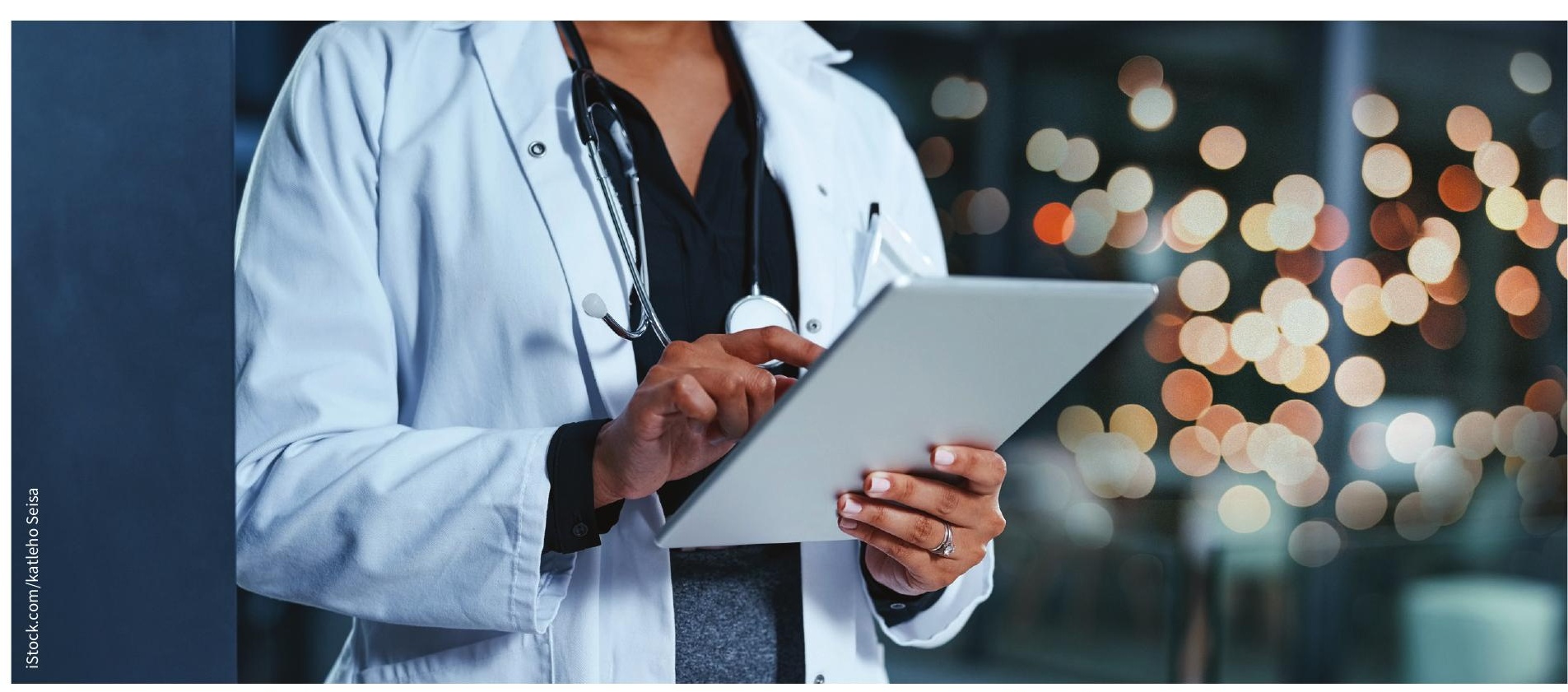

Medical trainees in the United States spend $43 \%$ of their shifts interacting with an electronic health record, found a recent study. 
that about a quarter of medical trainees' time was spent on direct patient care.

But according to Dr. John Neary, an internal medicine physician and associate professor at McMaster University, the older days shouldn't be glorified. In the 1994 study, one in three trainee shifts was a call shift longer than 24 hours, something now considered by many to be "not humane or sustainable." Those long call shifts alone would have driven up the percentage of direct patient care, he said.

Neary also wonders if the breakdown might be different in Canada, especially when it comes to EHR documentation. In the US, "there's an overdocumentation of normality that goes on," he said, in part due to "the medical-legal climate" and fear of malpractice suits.
As for multitasking, "the need to frequently switch tasks and properly account for a mental queue of work to be done, with items constantly being added to it and removed from it, is one of the most challenging things about acute care," said Neary. But multitasking is something that staff physicians do just as much as residents, he noted, so it's important for trainees to develop the skill of being able to switch back and forth quickly between tasks.

Dr. Steven Katz, director of the core internal medicine program at the University of Alberta, added that he expects a Canadian study would reveal more dedicated education time. "There is a lot of protected time for learning," he said, adding that residents in most pro- grams in Canada have a half-day of protected, pagers-off instruction a week. During a shift at his program, residents get "at least an hour, if not two, of formal teaching time, which is usually not interrupted."

He also suggested that direct patient care shouldn't always be seen as "better" than indirect patient care. "Advocating for patients is important; communicating with your team is important; following up on tests is important." How medical trainees in 2019 should divide their time depends on whom you ask. As Katz pointed out, "the question the study raises, and it doesn't provide the answers, is do we have the right balance?"

Wendy Glauser, Toronto, Ont. 